would appear premature to exclude them from research into the prodromal period.

The second issue relates to their sampling interval of one month, which they justify with reference to the literature. Birchwood et al (1992) have reviewed such studies, and more frequent measures have often been used. They report that up to $50 \%$ of schizophrenic subjects studied have been found to progress through a prodromal period to psychotic breakdown within four weeks. Many such prodromes would have been missed had a sample interval of one month been used.

Frequent prospective measures of a wide range of phenomenological experience are required before one is able to conclude that "a large proportion of psychotic episodes appear to occur without identifiable prior prodromal symptoms."

Birchwood, M., MacMillan, F. \& SMrTh, J. (1992) Early intervention. In Innovations in the Psychological Management of Schizophrenia (eds M. Birchwood \& N. Tarrier), pp. 117-129. Chichester: Wiley.

Chapman, J. L. \& Chapman, J. P. (1987) The search for symptoms predictive of schizophrenia. Schizophrenia Bulletin, 12, 497-503.

Gross, G. (1989) The 'basic' symptoms of schizophrenia. British Journal of Psychiatry, 155 (suppl. 7), 21-25.

KLOSTERKÖTTER, J. (1992) The meaning of basic symptoms for the genesis of the schizophrenic nuclear syndrome. Japanese Journal of Psychiatry and Neurology, 46, 609-630.

R. S. HOPKINS

Ashworth Hospital

Merseyside L31 $1 \mathrm{HW}$

\section{High-dose antipsychotic medication}

SIR: As a rehabilitation psychiatrist, I am frequently required to manage patients who are discharged from acute wards on doses of neuroleptics which are multiples of those I have been accustomed to use. I therefore initially welcomed the consensus statement on the use of high-dose antipsychotic medication (BJP, April 1994, 164, 448-458).

However, the consensus panel's advocacy of rapid dose reduction after acute treatment is based on too simple a model of courses during the recovery phase (Drury, 1992; Weiden et al, 1993) and is supported by a mistaken reference to Cookson (1987), who deals with relapses after a $50 \%$ reduction in high doses, and not on lower than routine doses as stated.

My own experience is of relapses, during gradual dose reductions in depot medication, at dose levels much higher than those on which the same patients were previously maintained well for long periods before admissions caused by medication refusal. I am puzzled by this pattern and wonder whether some form of rapidly developed drug tolerance may be involved (Sramek et al, 1990) or whether there are other so far unrecognised factors.

It is misleading and indeed potentially dangerous to include recommendations in consensus statements without either the clinical or the research data on which to base them. I would suggest that the consensus panel now systematically gathers and collates information about the effects of different strategies used by clinicians during this phase of treatment, as an initial step which may need to be followed by controlled trials.

Cookson, I. (1987) The effects of a $50 \%$ reduction of cis(z)flupenthixol decanoate in chronic schizophrenic patients maintained on a high dose regime. International Clinical Psychopharmacology, 2, 141-149.

DRURY, V. (1992) Monitoring recovery from acute psychosis. In Innovations in the Psychological Management of Schizophrenia (eds M. Birchwood \& N. Tarrier). Chichester: Wiley.

Sramek, J. J., Gaurano, V., Herrera, J. M., et al (1990) Patterns of neuroleptic usage in continuously hospitalised chronic schizophrenic patients: evidence for development of drug tolerance. DICP. The Annals of Pharmacotherapy, 24, 7-10.

WeIDEN, P., Schooler, N. R., SeVERE, J. B., et al (1993) Stabilisation and depot neuroleptic doses. Psychopharmacology Bulletin, 29, 269-275.

Goodmayes Hospital

David Abrahamson Essex IG3 $8 X \mathrm{~J}$

SIR: As psychiatrists in the adolescent subspeciality, we question the basis on which two main assertions are made in the consensus statement. First, it is asserted that "the natural history of early-onset psychosis is for the first few episodes to remit spontaneously". This corresponds neither with our clinical experience, nor with recent research studies (e.g. Werry et al, 1991; Gillberg et al, 1993). Typically, schizophrenia with an onset in adolescence follows a course similar to that in adults, and spontaneous remissions are rare.

Secondly, it is asserted that "High-dose antipsychotic medication should rarely be necessary in children and adolescents". Anecdotal clinical experience does not bear this out. Psychotic disorders in children and adolescents are not uncommonly refractive in their response to antipsychotic medication at standard doses (Green et al, 1992), and adolescents may tolerate adult doses with less risk of adverse side-effects (Garralda \& Ainsworth, 1987).

While we welcome the general spirit of the views expressed - it is important not to overmedicate children and adolescents, and expert treatment is always important - the views appear to us to be flawed in their details. Since no references are 